\title{
Software Process Modelling in EPOS
}

\author{
Reidar Conradi*, \\ Anund Lie, Espen Osjord, Per H. Westby, \\ Norwegian Institute of Technology, Trondheim, Norway \\ Vincenzo Ambriola, Univ. of Pisa and Udine, Italy \\ Maria Letizia Jaccheri, TecSiel, Pisa, Italy \\ Chunnian Liu, Beijing Polytechnic Univ., Beijing, P.R. China
}

Presented at CAISE'90, Kista, Stockholm, Sweden, May 8-10 1990.

\begin{abstract}
$E P O S^{1}$ is an instrumentable, kernel Software Engineering Environment (SEE). It consists of facilities for management of versioned products (configurations) through file-based workspaces attached to a versioned DBMS. EPOS will also manage the associated software development processes (tasks), being the subject of this paper.

The EPOS-OOER semantic data model can describe deriver tools, human actors, tasks and subtasks, projects, and triggers/notifiers; as well as normal software products. EPOS-OOER incorporates object-oriented ERA modelling, extended with tasking (PRE, POST, CODE) and simple type constructors (FORMALS, DECOMPOSITION). Customization is done through versioning of task types in project-specific workspaces.

Static task knowledge is expressed by types and subtyping, and is used for reasoning, planning, scheduling and execution of activities. Dynamic task knowledge is expressed by a versioned task network with a horizontal (temporal) and a vertical (decomposed) dimension. Tasks are connected to products by normal relationships.

Keywords: Object-Oriented ERA Model, Planning, Software Configuration Management, Software Process Management.
\end{abstract}

*Address: Division of Computer Systems and Telematics (DCST), Norwegian Institute of Technology (NTH), N-7034 Trondheim, Norway. Phone: +47 7 593444, Fax: +477 594466, Email: conradi@idt.unit.no.

${ }^{1}$ EPOS, Expert System for Program and ( $" \mathrm{Og}$ ") System Development, is supported by the Royal Norwegian Council for Scientific and Industrial Research (NTNF) through grant ED0224.18457. Do not confuse with the German real-time environment of the same name [Lem86]! 


\section{Introduction}

Experience with SEEs indicates that an open architecture is crucial to avoid straitjacketing effects. Both new and old tools must be accommodated, and relevant company or project policies should be explicitly stated, enforced, reasoned about - and changed. This puts high demands on the expressivity and flexibility of the underlying formalism.

A software product is described by many interrelated and evolving software components. A software configuration management (CM) tool is therefore needed to control the evolution of such systems. Most CM tools are marginally aware of the underlying software processes and their management (PM). Such a development process is often described in terms of the product (what), while the process (manual or automatic) can specify how and why a version of a product is constructed. In other words: the CM and PM areas should be integrated. Much work has recently been spent on PM in order to understand, model, execute ("enact") and record the operations performed on a product. Such operations or processes range from simple tool invocations to high-level design and project-related activities performed by human actors. A perpetual argument in the PM area has been human creativity vs. automation [Leh87].

Several generic or meta-models for PM have been introduced. These can be instantiated or customized into a more specific process model, e.g. a waterfall, spiral or project-specific model. This puts high demands on the dynamics and generality of the underlying type system.

The EPOS PM approach is to integrate:

- Static process programming as in ARCADIA [TBC*88] and IPSE 2.5 [OR86].

- Dynamic (sub)contracts as in ISTAR [Dow87].

- Rule-based reasoning as in MARVEL [KF87] and ALF [B*89],

- Networked tasks with dynamic triggering à là OSMOSE (Petri net model) [DGS89], and to some extent PCMS [HM88] and NOMADE [BE87].

- Subtype refinement as in Process-Oriented CM [BL89].

Some more high-level structuring is needed, but we are only describing the kernel facilities and basic type system.

The ensuing sections of this paper are as follows. First the EPOS architecture and basic CM model of EPOS are summarized. Then the overall PM model and the associated Activity Manager are presented. Then follows a more formal treatment of our object-oriented ER model, EPOS-OOER, with emphasis on PM-relevant type properties, type constructors and project-specific versioning. Lastly, some present problems and ideas on future work are given. 


\section{EPOS Background}

\subsection{EPOS Architecture}

The main EPOS components are:

- A semantic data model, EPOS-OOER.

- An advanced $C M$ system, p.t. on top of the INGRES relational DBMS [SWKH76].

- An Activity Manager and Planner for PM support. The Activity Manager includes a Builder.

- A set of EPOS support tools, such as a Product Editor and a Maintainer's Assistant.

- A User Interface, based on the X Window System.

- Local, checked-out workspaces or configurations (files, databases in special formats), accessed by misc. programming tools.

EPOS will run on Unix workstations, using INGRES, $\mathrm{X}$ Windows, $\mathrm{C}++$, and Prolog. See [CDrG*89] for more details.

\subsection{Change-Oriented Versioning and Related CM}

We have adopted the change-oriented model (COM) to versioning [Hol88] [LDC*89] [LCD*89]. Here, a functional change involving several (related) components is described by a single, global option. COM resembles and generalizes conditional compilation, and is fundamentally different from more conventional, version-oriented models (VOM). Most most database (DB) items can be uniformly versioned from a technical point of view. Some COM concepts are:

- Option: Essentially a boolean variable to describe a functional change (external property), such as MachineSun or BugFixCommandA. It is not an object attribute as in VOM, rather a non-versioned(!) entity with its own attributes: DateTime, Validity, Name, etc.

- Validity: Boolean expression over options to describe valid combinations of options. It corresponds to attribute constraints in VOM.

- Fragment: Basic information item, such as a relational tuple or a text line.

- Visibility: Boolean expression attached to each fragment of Versionedobj instances.

- Version: It consists of all fragments where the visibility evaluates to True for the given version-choice. A version is not an instance itself, but the result of a functional evaluation!

- Version-choice: A set of (option,value) bindings to describe which version an application task wishes to see. A version-choice is complete if a unique version of the DB is produced.

- Version-description: High-level DB query, that maps to a low-level version-choice, which defines a specific version-view or version of the DB.

- Product-description: Tuple of (Root objects, ER types), that maps to a product-view or product closure on the DB. The product is described by SysBody and similar entity 
types. Note, that all DataEntity instances have a long Contents field, represented by an external file.

- Config-description: Generic (Product-description, Version-description), that maps to a config-view closure on the DB.

- Specific, primary configuration: a config-view to be used as a workspace by developers or tools.

- Specific, derived configuration: can be produced by the Builder as Deriv-Config := Build( Product-view(Version-view( DB ))).

- Change job: An edit-update task that controls a long transaction associated with a workspace, thus linking CM to PM.

- Ambition: A non-complete version-choice associated with a change job. It identifies the set of version-choices where new changes are going to be visible.

To interface old tools, the only viable solution is to check-in and check-out local workspaces of files and related DB information. This corresponds to the copymodify-merge paradigm in NSE and PACT [Sun88] [Bul87]. An example of two coordinated workspaces is shown in figure 1 . Here, workspace $\mathrm{WS}_{1}$ belongs to Project $\mathrm{P}_{C}$. This is a subproject of project $\mathrm{P}_{A}$ that controls workspace $\mathrm{WS}_{2}$. Each

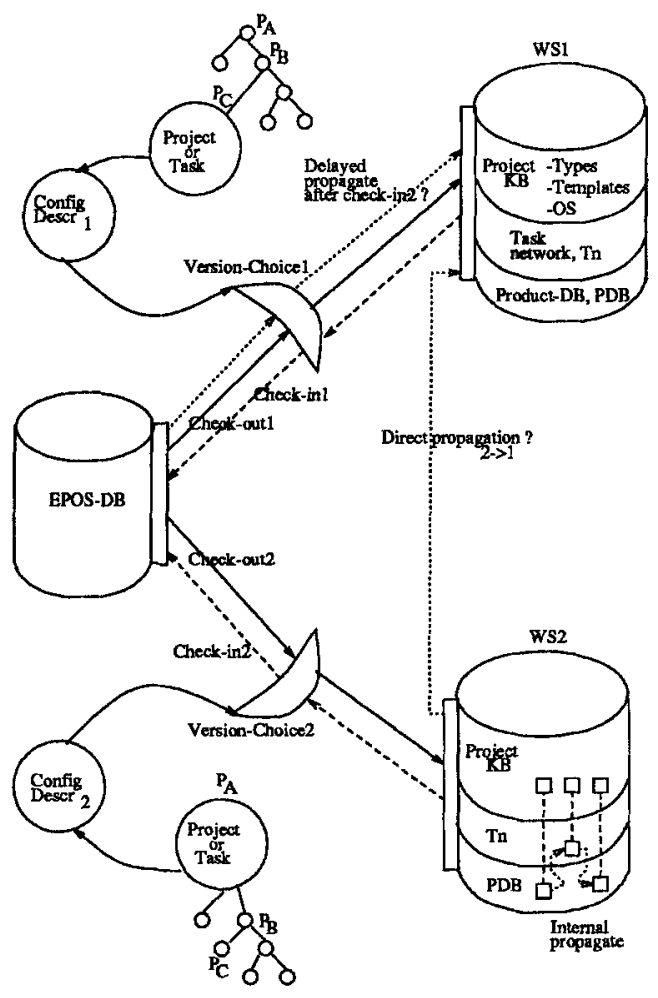

Figure 1: Central DB with checked-out workspaces, $\mathrm{WS}_{i}$

workspace or configuration is controlled by a change job and its configuration 
description. A workspace is divided in three: product DB, task network, and project knowledge base (KB, with types and various other descriptions).

\section{$3 \quad \mathbf{P M}$ in EPOS}

\subsection{General Demands for Generic PM Support}

Our goal is a common, system-interpretable formalism to describe software development processes. The model must cover:

- Deriver tools: their description (pre/post-conditions, inputs/outputs, tool switches) and aggregation.

- Human actors in an open-ended way.

- "Active" relationships to express change propagation by triggers.

- Chained tasks for horizontal life-cycle phases, revision lines, or derivation graphs.

- Subtask hierarchies to describe vertical work decomposition.

- Complex interactions between tasks and tools/users.

- Special project tasks to control workspaces with project-specific information (e.g. types).

\subsection{A Survey of the PM Model}

Our activation mechanism is coarse-level and mainly descriptive, based on PREand POST-conditions in types. Such conditions seem to flexibly express the activity rules for PM. They are also well-suited for static, forward and backward reasoning without executing the CODE.

The CODE associated to a task instance is responsible for causing its POSTcondition to become True, and thus cause ("fire") other PRE-conditions to become True etc. The POST/PRE coupling therefore serves as a dynamic synchronization mechanism. The CODE of a task may re-execute, repeating the PRE/POST pattern above.

The experience with unrestricted "firing" of unbound rules and triggers ${ }^{2}$ in databases, AI applications, and syntax editors [HN86] made us sceptic to such solutions. In our case, direct task communication is limited to relationship-connected neighbor tasks (4.5.1), and can thus describe traditional message passing and notification. Note the analogy with Petri nets [Rei85]. Note also that task execution can occur at any network node, not only at the leaves - cf. ISTAR. This distinguishes the network from a finite state machine, where only one node at a time is active.

\footnotetext{
${ }^{2}$ We may, of course, consider a task with a PRE-condition and an imperative CODE as a "trigger", with syntax IF - THEN - . Note also, that the EPOS task type corresponds to a conventional AI rule.
} 
Dynamic subtask creation allow for considerable freedom in organizing the software work, and alleviates the limitations of static CODE in instantiated tasks. There are FORMALS and DECOMPOSITION properties (constraints) to regulate the structure of the task network.

\subsection{The Activity Manager and Planner}

Management of task types and instances is done by the EPOS Activity Manager $(A M)$. and its associated Planner [Mul89] [LC89]. High-level or more complicated tasks must be delegated to human actors. Generally, we will have a high-level goal to achieve, and a product $\mathrm{DB}$ and a project $\mathrm{KB}$ as the starting point.

The relations between the Activity Manager and types, tasks and products are shown in figure 2:

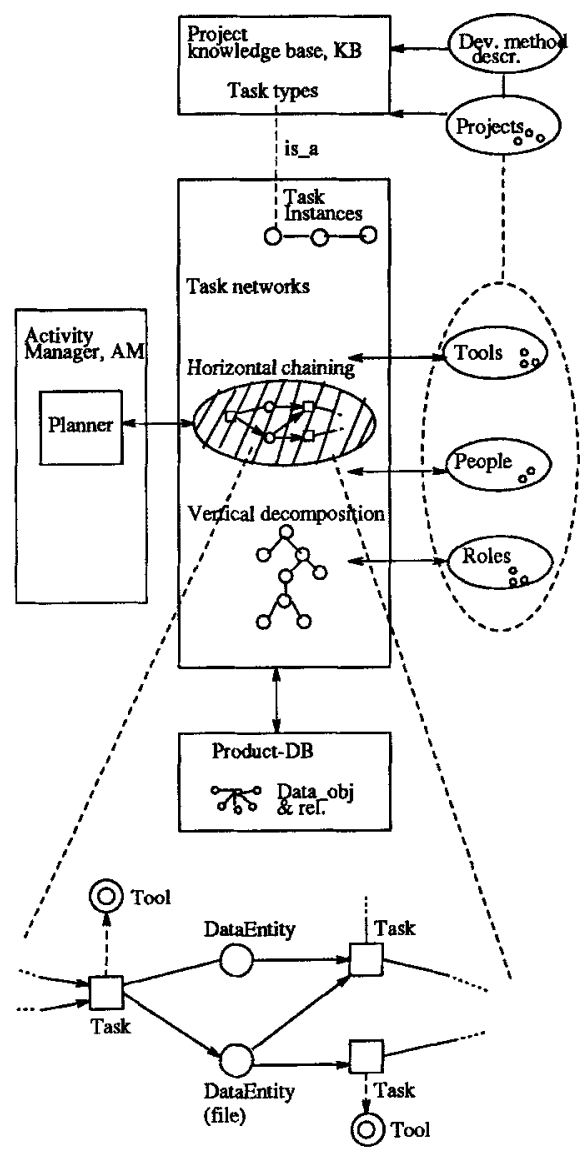

Figure 2: AM and data 
The Activity Manager consists of:

- A Type Manager (TM) to construct and modify specific TaskEntity subtypes. The TM cooperates with a project manager or another meta-user.

- An Instance Manager (IM) to dynamically create, edit, and delete task instances; and to manage horizontal and vertical composites of such. The FORMALS and DECOMPOSITION information from task types will be used to automatically construct new and type-validated task structures.

The IM cooperates with the Planner or a user to handle simple tasks (tool activations), and with a meta-user for more complex tasks (e.g. projects).

- An Execution Manager (EM) to interpret, execute, control and record task instances - while obeying the static task knowledge. E.g., tools must be called correctly, with dynamic checking of parameters if necessary.

The EM cooperates with tools, users, the Builder (an EM component), and the Planner. Possible error situations must be monitored for alternative actions, and may include replanning and re-execution.

The Planner will:

- Offer product-level assistance such as construction of the empty derivation graph - a plan - for the Builder, cf. [HC88]. The plan may be incomplete, and a TaskState attribute of the planned tasks may reflect this.

- To some extent offer project-level assistance about task or work decomposition, work plans, etc.

- Utilize static, method-specific type knowledge about legal task communication and combination patterns. This is expressed by PRE, POST, FORMALS and DECOMPOSITION properties. This knowledge will be combined with information from the product DB. Only the non-temporal aspects of PREand POST-conditions will be considered, excluding comparison of DateTime timestamps, or scheduling rules like <Compile between 24:00 and 06:00>.

- Replan upon execution failures.

- Assess the impact of changes.

- "Learn" by putting generalized PM types back into the KB.

The Planner will borrow ideas from MARVEL, the AGORA PLANNER [BLA88], the domain-independent IPEM [AS88], and case-based planning.

\section{EPOS-OOER, The Semantic Data Model}

A common, semantic data model for $\mathrm{CM}$ and $\mathrm{PM}$ is sought, since there is much interaction between the two areas. EPOS-OOER represents a unification of ER [Che76] and object-oriented (OO) modelling, and allows general subtyping. The use of low-level pointers instead of general relationships in 00 models has been remedied [Rum87]. From semantic network models we have been inspired by uniform handling of entities and relationships [TL82]. 


\subsection{Available Type Properties}

Multiple subtyping is specified through SIMULA-style prefixing. We will not define the schema notation formally, as the examples should be self-explanatory. The repertoire of type properties is explained in the following sections.

\subsubsection{DOMAINS and ATTRIBUTES}

Attributes belong to given domains, and represent passive variables or active $P R O$ Cedures (methods). Domains are declarable, and include scalars, text strings, pointers (REFs, see below), PROCs, and pre-defined ones like Bool. A REF-value is the value of the $0 b j I d$ attribute of the referred object (see RootER type below). REFs are restricted to non-PersistentObjs, temporary PROC parameters, and access functions for CONNECTION data (4.1.2). In task types externally callable PROCs are constrained to functions without side-effects, i.e. functional attributes. Initialization is provided by = <init-value . CONST means read-only or systemmaintained variables.

Inheritance means that the supertypes' global attributes and domains are inherited into the subtypes. Multiple names in the supertypes are resolved by bottom-up, left-right search. A local name will always hide or overload the global one.

An example of a type definition with domains and attributes is:

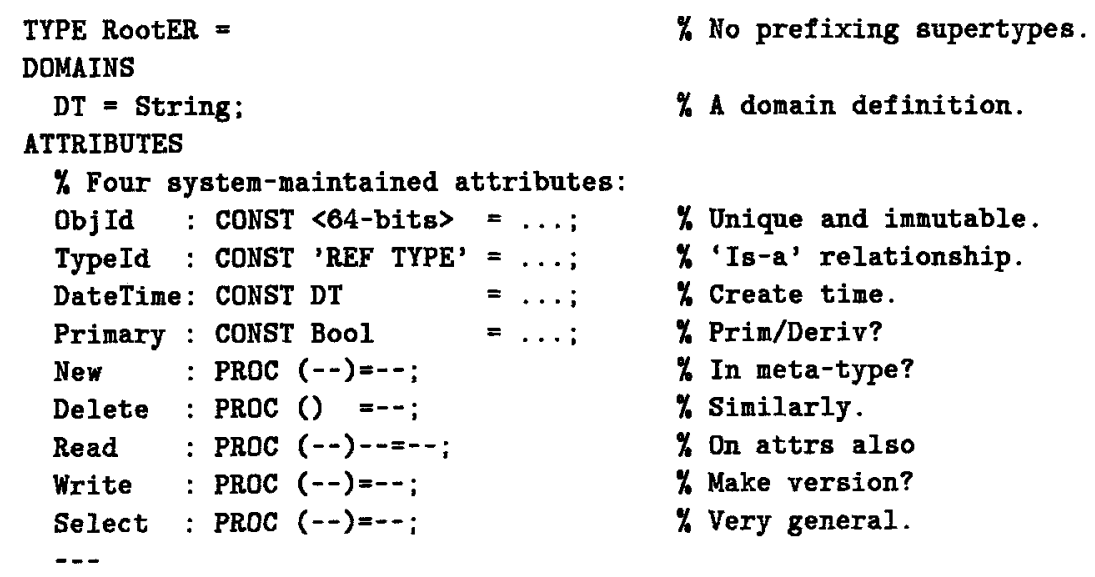

END-TYPE RootER;

\subsubsection{CONNECTION}

Types with no CONNECTION property are implicitly called entity types. Inversely, a CONNECTION implicitly identifies a binary relationship type, that connects two entity types ${ }^{3}$. Only single type inheritance is allowed at the user

\footnotetext{
${ }^{3}$ In ER terminology, roles should have been used here. - And the debate whether relationships should have identity and TypeId or not, is still going on!
} 
level, starting from the Relationship type below. Inheritance may constrain cardinalities and related entity subtypes, and may rename the access functions.

An example of a basic relationship type is:

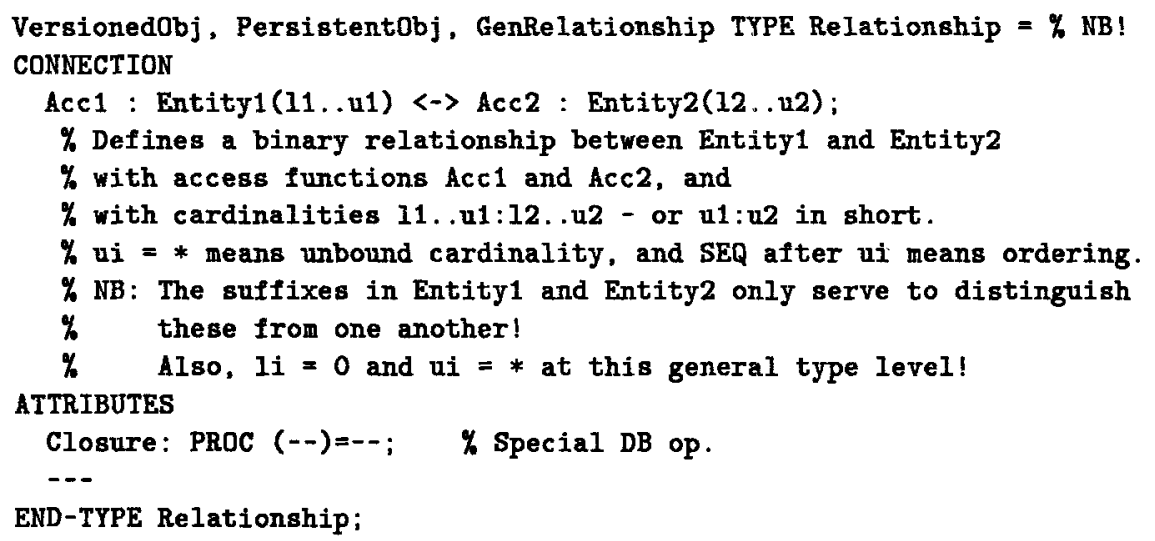

Named access functions (Acc1 and Acc2 above) are implicitly available to the "related" entities, but formally defined in the connecting relationship type. Assuming a cardinality of $*: 1$, Acc1 could be defined in Entity2 with domain 'SET(REF Entity 1)'. With a cardinality $1: *$, the domain of Acc1 could be 'REF Entity1'. "CONNECTION data" may be stored inside the related DB entities as pointers or sequences of such for reasons of efficiency.

A short-hand notation for simple relationship types, implicitly prefixed by Relationship is offered:

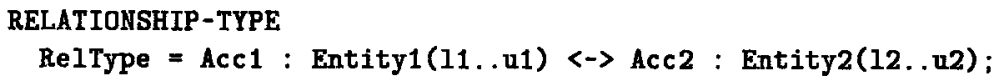

\subsubsection{CODE}

This is a piece of program code expressed in an imperative programming language, executed by the Activity Manager. CODE is primarily used in TaskEntity types, but may be used for initialization of non-tasks. However, such instances are not "active" tasks in the OO sense.

The CODE language is not concurrent, as all triggering is implicitly expressed by PRE- and POST-conditions. The CODE language is a restricted Unix shell language. STOP means task termination. Shell variables such as $\$<$ name $>$ can be accessed. Into (AccFunc, $X$ ) means insertion of $X$ into a relationship. INNER means execution of the subtype's CODE. It will be appended in the CODE part, if missing. Type inheritance implies concatenation, using SIMULA's INNER mechanism. 


\subsubsection{PRE- and POST-conditions}

As mentioned, a task waits for its PRE-condition to become True before (re)activation of its CODE part. After each activation, the POST-condition must be fullfilled. PRE and POST are intended for task types, but can be envisaged as initialization and termination constraints elsewhere. Type inheritance is by conjunction $(\wedge)$, starting from True in the TaskEntity type.

PRE- and POST-conditions are formulas in first-order predicate logic, with the following predicates and functions:

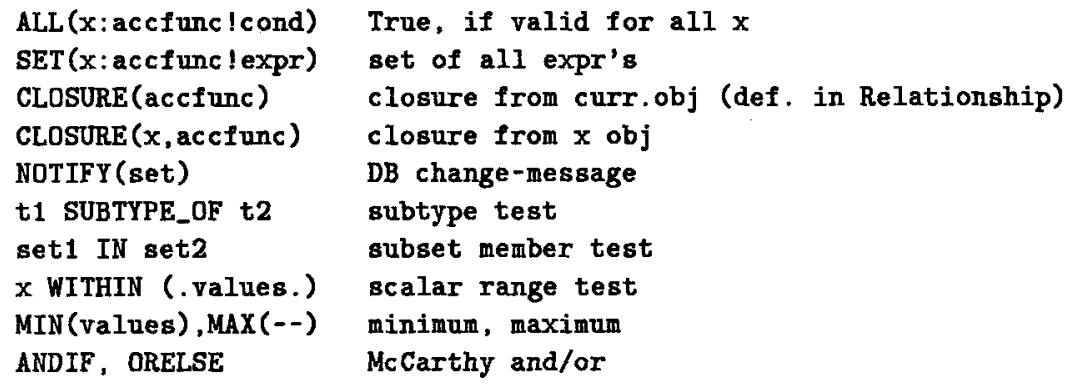

For technical reasons, the PRE-condition is split into two parts: PRE_STATIC used by the Planner and PRE_DYNAMIC used by the Execution Manager. We have not yet found it necessary to split up the POST-condition similarly.

The PM types (4.5) contain examples of PRE- and POST-conditions.

\subsubsection{FORMALS and DECOMPOSITION constructors}

Both specify simple type templates, and are restricted to TaskEntity type and subtypes. Type inheritance rules are as for attributes, although we have considered various schemes of subtype-constraining semantics [CW85].

FORMALS constrains the indirect, horizontal chaining of tasks, i.e. the legal types of actual task parameters. These parameters are expressed by the GenInputs and Genoutputs relationships between TaskEntity instances and their inputs and outputs (4.5.1).

An example of a FORMALS specification is:

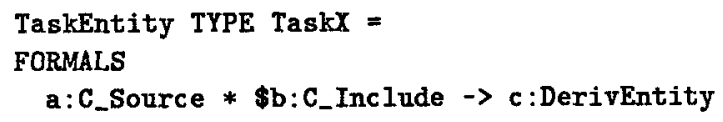

This means that TaskX instances take one input of type C_Source and a variable number of inputs of type C_Include (because of the starting \$-sign in the parameter name), and produce one output of type DerivEntity - or subtypes thereof for all these types. All parameter types must be subtypes of Entity, i.e. no relationships can be processed by tasks. The default FORMALS specification is: 
\$in:Entity $\rightarrow$ \$out:Entity

i.e. no constraints at all. CHECK_IN_FORMALS and CHECK_OUT_FORMALS predicates are available for use in PRE/POST-conditions, see Sec. 4.5.1.

DECOMPOSITION constrains vertical task breakdown, i.e. the SubTasks relationship between parent and children tasks (4.5.1).

An example of a DECOMPOSITION specification is:

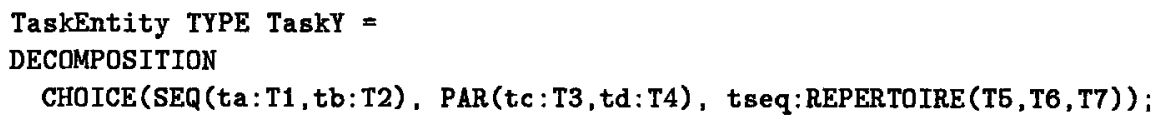

This means that possible children of TaskY instances may be either instances of $\mathrm{T} 1$ and $\mathrm{T} 2$ executing in sequence, instances of $\mathrm{T} 3$ and $\mathrm{T} 4$ executing in parallel, or any number of instances of T5/T6/T7 executing in parallel (PAR is implicit). The default is REPERTOIRE (TaskEntity), i.e. no constraints.

The Activity Manager and Planner uses DECOMPOSITION for automatic generation of children tasks after creating a parent task, and so on. To avoid redundancy with Into(Children, NEW TaskEntity (...)) in the CODE part, the DECOMPOSITION should be empty, if the parent task explicitly generates or kills its children tasks. In case of a SEQ specification, synchronization of children tasks will be checked and possibly enforced by the Planner.

\subsubsection{EXECUTABLE}

This identifies the logical name of the associated OS-tool of a deriver task. Such a tool can be considered an "external" PROC, possibly shared by many similar task types. Upon workspace initialization, a "soft link" between the type object(s) and the selected Executable instance will be established.

\subsubsection{INVARIANT}

This is a formula in first-order predicate logic, specifying an assertion over certain DB instances. It should always be True. Type inheritance is by conjunction $(\wedge)$. Sec. 4.5.5 contains an example of an INVARIANT.

\subsection{Pre-defined Types}

A type semi-lattice of some of the pre-defined types is shown in figure 3 , with the system-defined ones above the dotted line:

For a real project, all the main types have much more subtypes than indicated. 


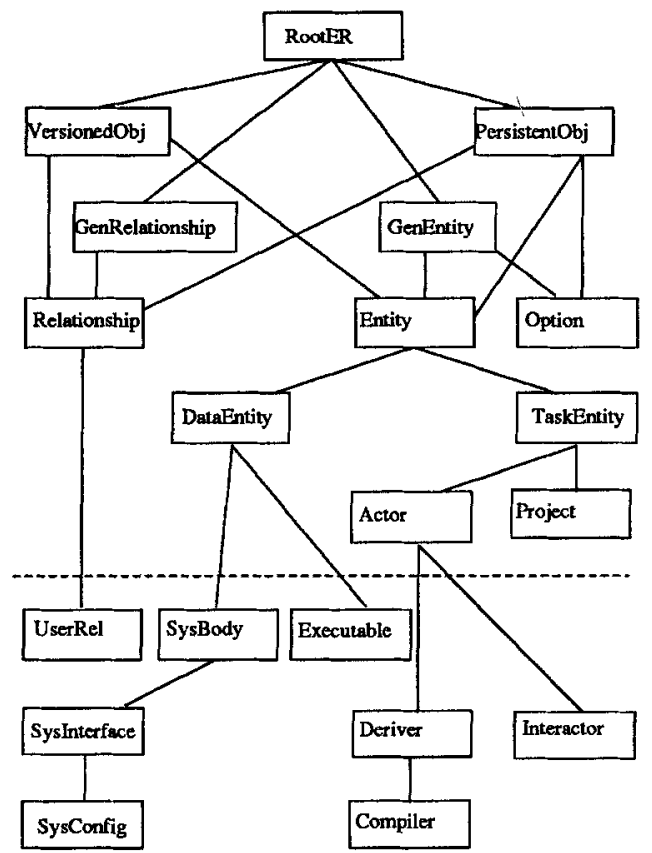

Figure 3: EPOS type semi-lattice

\subsection{General Comments on EPOS-OOER}

DB instances of any type can be created, by NEW Type (List of <AttrName = Value >). Entity instances are often called "objects".

The types are represented as normal DB objects or TypeDescrs (4.4), available for dynamic interpretation. There are no meta-types.

The non-PROC ATTRIBUTES and CONNECTION part of EPOS-OOER is called the $C M$-part. The rest is called the $P M$-part, since such properties are stored at the type level and are implemented by the Activity Manager. This PM-part can be versioned (4.6).

Note, that EPOS-OOER is not quite "unified": There are limitations on the use of pre-defined types as supertypes. E.g. Persistentobj and Versionedobj (2.2) mostly serve as keywords, GenRelationship subtypes are restricted to simple inheritance, and option cannot be used as a supertype at all. Type properties beyond domains and attributes are only relevant for TaskEntity subtypes.

An alternative conceptual base would have been more specialized type categories - DATAENTITY, RELATIONSHIP and TASKENTITY - and a more closed data model. 


\subsection{On Task / Tool modelling}

The OO ERA modelling in EPOS-OOER follows conventional patterns. The problem has been the modelling of tasks and tools, where the solutions have changed fundamentally over the last year. We will repeat the relevant arguments and rationale for the uninitiated reader.

The main PM type is now TaskEntity (4.5.1). A TaskEntity instance describes a potentially active process or task. Its CODE part may execute its own program, and indirectly that of its children. The low-level, more specialized Deriver tasks may directly call passive OS-tools, so-called Executables, through a procedural envelope with given file parameters. Static tool aggregation is done by DECOMPOSITION of associated task types. Versioning of OS-tools is done by the normal CM mechanisms, and versioning of task types is explained in Sec. 4.6.

We have also had problems in modelling formal parameters and task/tool templates. Both these imply type-level "relationships" or constructors to express constraints, which are not easily expressible in an ER framework:

- Formal parameters were initially expressed by ad-hoc ProcInputs and Proc Dutputs "meta-relationships" to connect a TaskEntity type with its legal input and output types. The type information of such formal parameters could then be matched against the types of the actual parameters.

It remains a problem to compactly express shared formal parameters between similar deriver tasks. We may need a separate signature type ("arrow" type) for this.

- High-level templates for task/tool aggregates and task networks may assume type composites at the meta-level.

Instead of having a more sophisticated type apparatus on the meta-level, we have introduced the FORMALS and DECOMPOSITION properties. However, we have no separate Template type to describe more generalized type composites.

FORMALS and DECOMPOSITION are internally implemented by systemmaintained "shadow types", TypeDescrs, at the instance level. Such TypeDescrs facilitate, in principle, any kind of high-level typing through arbitrary type relationships or constructors ${ }^{4}$.

Some more technical arguments have been:

- An EPOS Deriver task is an abstraction of an activation of a real OS-tool. Such an OS-tool is called by issuing a command line (a script) to the OS, with proper tool name, tool switches and file parameters.

- Tool envelopes are needed to hide OS details and to provide project instrumentation and error treatment.

- Tools cannot be expressed as traditional OO "methods" (PROCs) in such types. This is because the tools may be shared by several task types and

\footnotetext{
${ }^{4 E P O S-O O E R}$ only supports "Subtype_Of", FORMALS and DECOMPOSITION type constructors.
} 
possibly independently produced and versioned by the surrounding OS - $\mathrm{cf}$. EXECUTABLE property and next item.

- Tool switches cannot easily be modelled by traditional parameters, due to varying number and special semantics. E.g., some switches cause extra output to be produced, so that different "deriver variants" must be defined with appropriate FORMALS ${ }^{5}$. Centralized control over default tool switches is also desirable.

- Tool aggregates are needed to hide details, using DECOMPOSITION to express super-tools.

- Lastly a reminder: We do not want to model all the "hairy" semantics (to put it mildly!) of the OS-tools. Only the essential parts for basic tool management need be covered.

There are three different task/tool breakdowns, all N:M:

- The static type semi-lattice.

- Static task/tool type aggregates through the DECOMPOSITION constructor.

- Dynamic decompositions of task instances through a SubTasks relationship, obeying the DECOMPOSITION constraints.

\subsection{The main PM Types}

\subsubsection{The TaskEntity Type}

TaskEntity has an Actor subtype (4.5.2) to execute an interactive or automatic tool, a Project subtype (4.5.5), and more specialized subtypes.

As mentioned, the CODE in a task instance is executed when the PRE-condition evaluates to True. A task serves as a coroutine - with an implicit, embedding loop around the outermost CODE part. The task execution environment is assumed to be cheap - probably co-routines in the Activity Manager, plus forking of real OS processes to execute OS-tools.

The Taskentity definition is:

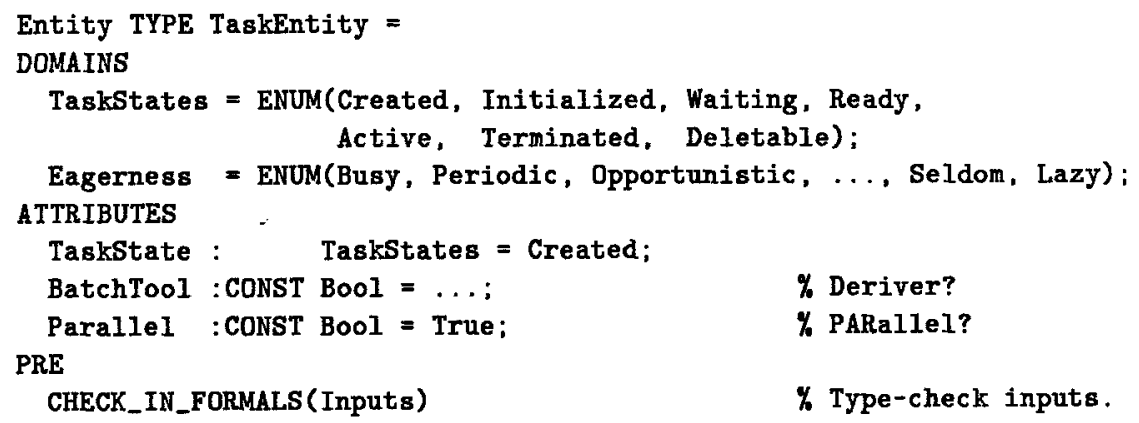

\footnotetext{
${ }^{5}$ Indeed, the Unix CC-compiler may be used both as a pre-processor, compiler, linker, assembler, ... - and with different FORMALS within these categories!
} 
CODE \% Coroutine:

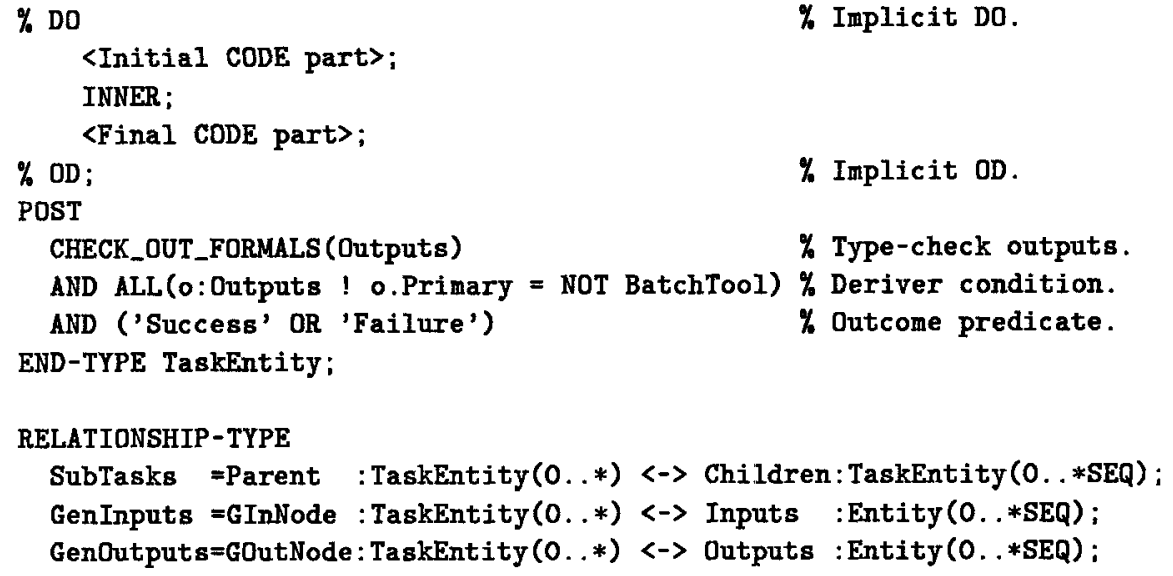

Name attributes of GenInputs and Genoutputs actual parameters are omitted for sake of clarity.

\subsubsection{The Actor Type}

Actor is a trivial TaskEntity subtype; not shown. It has two subtypes, an Deriver (4.5.3) and Interactor (4.5.4).

\subsubsection{The Deriver Type}

As mentioned, the Deriver type represents a deriver tool. Its CODE part is a shell script to prepare, activate, control, and record an OS-tool activation. This is done through "INNER"-subtyped envelopes of Pre/Post-Actions. Such actions may include "invisible" control and accounting functions, as in GENOS [GEC87]. The logical name of the OS-tool is given by the EXECUTABLE property. Default tool switches will be provided from the global CurrProject. "Crucial" tool switches, affecting the FORMALS, are contained in a local DeriverSwitches attribute. This attribute and the matcher PROC attribute (see 5.1.1) will be redefined by subtypes.

A Deriver definition with a busy rebuild rule is:

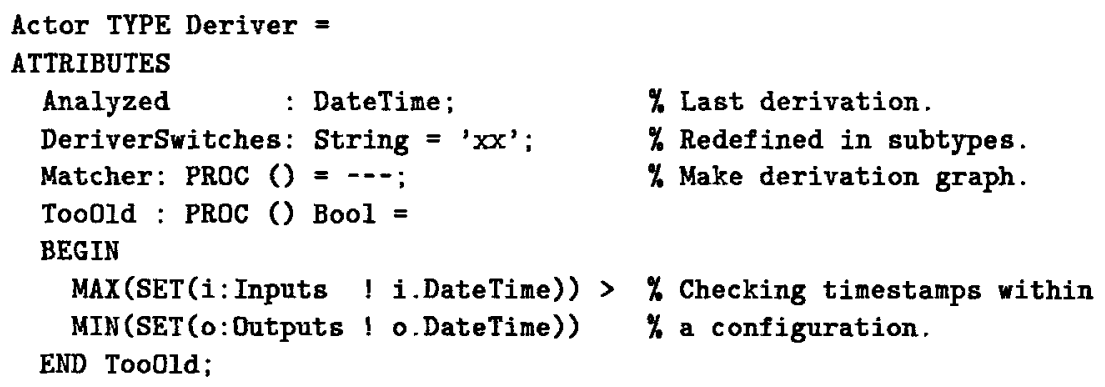


Too0ld() AND CurrProject.Rederiv_Policy ()$=$ Busy

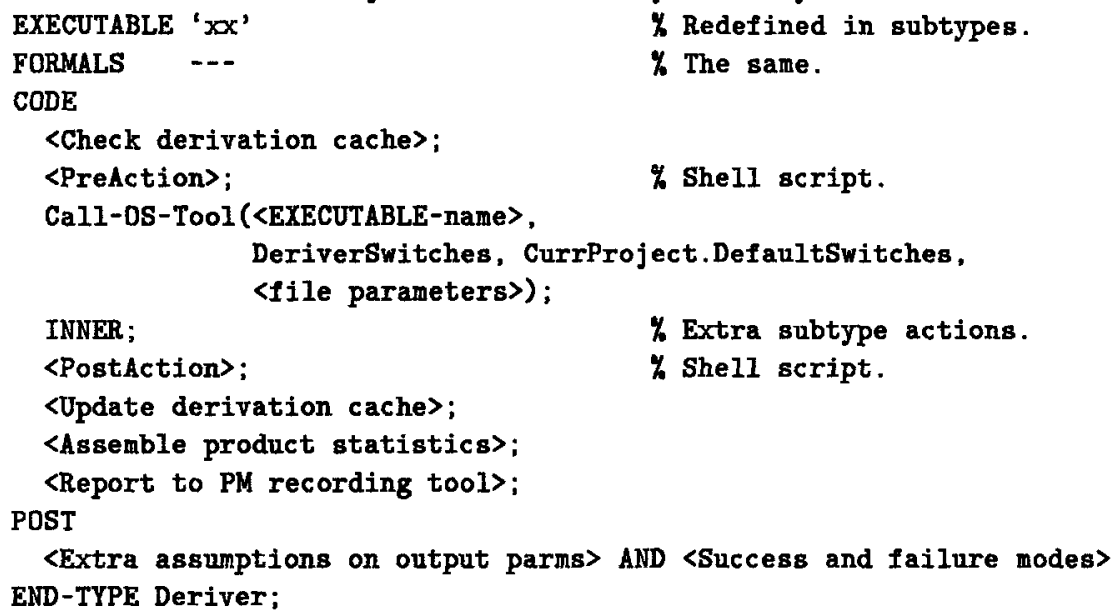

A coarse description of success and failure modes must be supplied. Note, that each project may define its own rederivation policies (PRE-conditions) or CODE parts.

A deriver is typically a compiler, link editor, text formatter etc. We can envisage a Compiler subtype, with a CC-Compiler subtype of this etc.

\subsubsection{Interactor Type}

Interactor is an Actor subtype representing an interactive tool activation, coupled to a Role instance. The Interactor definition may look like:

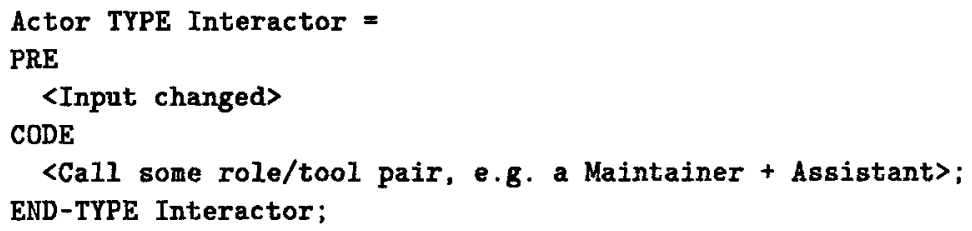

A Role represents a "canonical" person, emphasizing authorization, job position, and general project attachment. The Role will again be connected to specific Person(s), having responsibility of certain software components.

\subsubsection{Project Type}

Project is a TaskEntity subtype to emphasize the productive aspect. A project specifies a project $K B$ connected to a workspace. A new project instance, e.g. CurrProject, may be created in the workspace of its parent project. The new project will inherit the parent's workspace, and in addition create a nested 
workspace of its own. In principle, the project $\mathrm{KB}$ can be changed on-the-fly! See Sec. 4.6 for binding mechanisms to achieve flexible project tailoring.

The project KB should contain the following information:

- A config-description (2.2) for the current DB transaction in the local workspace. This may include a traditional DB view, with rights and capabilities.

- A coarse OS description: specific or low level OS-tool information, such as file bindings, environment flags, default OS-tool set including tool switches (e.g. -I <directory_name> to compilers), ...

- Subprojects, and allocation of persons and resources - as in ISTAR.

- Project-specific rules and policies, through various PM types, task/tool templates, invariants, project-pervasive attributes, ...

Ex. Project policies for CurrProject $=$ ProjectX.

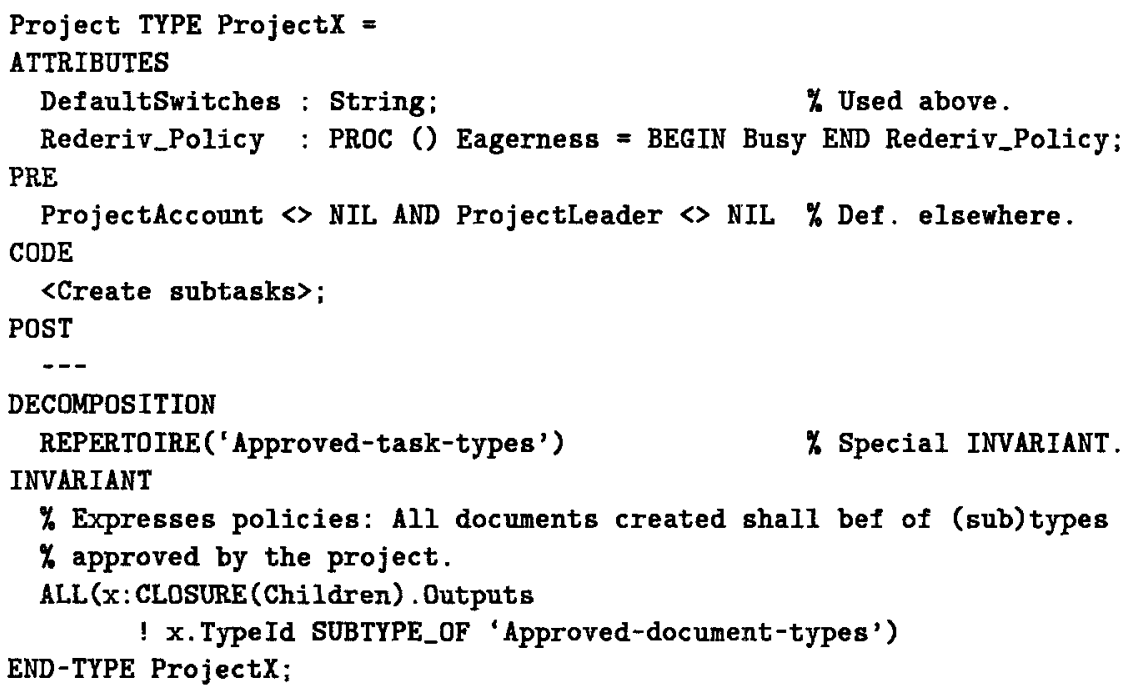

\subsubsection{Executable Type}

This is a DataEntity subtype, and specifies BinaryProgs, Shel1Scripts, or other OS-tools.

\subsection{Project Customization of Typing}

Some possible binding mechanisms are:

- Static inheritance in the task type hierarchy, i.e. project-specific subtyping. This may lead to a proliferation of subtypes and mutual constraints on correct subtype selection, cf. option validities and figure 4 . 
- Type parameterization and instantiation of generic project types, as an alternative to subtyping.

- Project versioning. a generalization of subtyping, which is easy to achieve in our COM model from a technical point of view. However, we want essentially non-versioned types/DB-schemas to prevent a semantic explosion of sub-universes in the DB [SZ86].

- Dynamic inheritance in the task instance hierarchy, or along any given relationship.

- Dynamic instrumentation of the Activity Manager through special couplings to CurrProject, cf. CurrProject. Rederiv_Policy used by the Builder.

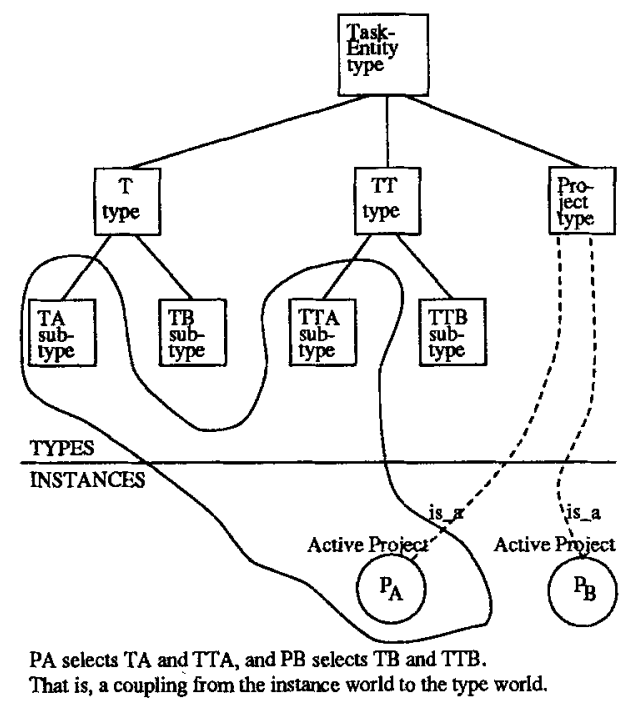

Figure 4: Consistent choice/version of subtypes

The EPOS solution is to use the system-maintained TypeDescr instances (4.4) to express limited type versioning within the workspace of a project, i.e. of the PM-part only. This guarantees some minimum DB stability.

A comment on the EPOS model of type versioning: It may seem primitive to let a set of boolean or scalar options "parameterize" our type system. However, we want to version an entire collection of types and other control information at the same time. Alternative approaches with sophisticated type parameters in addition to subtyping (cf. Eiffel [Mey88]) are more complex but still insufficient. In contrast, our scheme uses the existing versioning and workspace mechanisms in EPOS, i.e. it has a low human and computer cost. 


\section{Applications of the PM Model}

\subsection{Active Relationships, Derived Objects and the Builder}

\subsubsection{Active Relationships and the Derivation Graph}

An intuitive modelling of "active" relationships is to associate triggers (rulecoupled tools) with relationship types. Since we do not allow $n$-ary relationships with possible task decomposition, we must insert extra task entities between the "N:M"-related objects, see next paragraph.

The conversion from a dependency graph with "pure" relationships to a derivation graph with inserted task nodes is language-specific. It is taken care of by the matcher PROC in the Deriver subtypes. For instance, GenInputs in a derivation graph must represent the transitive closure of the relevant inputs for programming languages like C, Pascal and Fortran (large, but shallow graph). That is, the Deriver step to compile a C-program X.c will require the "body" file X.c, the "interface" file X.h, and all .h files that these two files transitively include. This closure is not necessary for languages like Ada and Modula, having separately compiled interfaces (smaller, but deeper graph).

\subsubsection{Derived Objects}

Derived objects are different from primary objects. A derived object of type Entity is stored as a versioned, functional DerivResult "attribute" in an instance of Deriventity type (not defined here). This instance represents a possibly empty version group of derived objects. Different tools operating on the same input objects must be described by different derived objects and derivation graphs.

When requested by an application, the versioned DerivResult attribute may be regenerated, using the available derivation graph. This corresponds to lazy build. The DerivResult attribute identifies the derived output, its inputs, and tool version and tool switches used. There is an accompanying, versioned DateTime attribute.

The set of non-empty versions of the DerivResult attribute can be treated as a global cache of derived objects, and is subject to user policies for deletion; see ODIN [Cle88]. It is important to share attribute versions between configurations - i.e. smart recompilation! - by "increasing" attribute visibilities [Lie89].

\subsubsection{The Builder}

The Builder operates in the current or LOCAL workspace (2.2). It is really a part of the Activity Manager, which also has created or planned the derivation graph of task objects. An example of a derivation graph is:

The Builder will generate a complete, derived configuration - upon explicit re- 

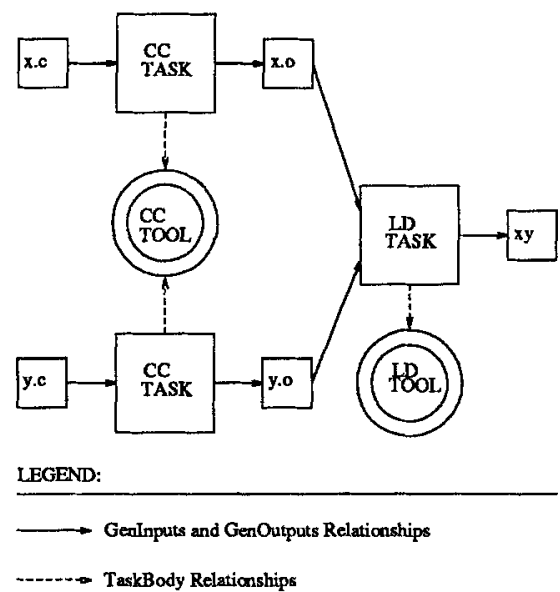

Figure 5: A derivation graph

quest (lazy, backward chaining), or when triggered by project-specific rules (busy, forward chaining). Error handling is difficult, e.g. to interpret compilation errors sensibly. Suppose that we want to nightly rederive the outdated objects, caused by DB-changes in the LOCAL workspace:

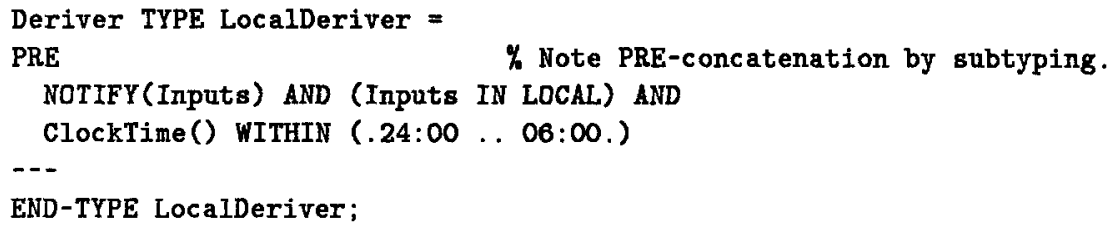

Such a policy could also have been defined in CurrProject.Rederiv_Policy (4.5.5).

\subsection{Subtasking and Task Sequencing}

The task-subtask hierarchy, as illustrated in figure 6, covers many different purposes.

Projects fit nicely into the task hierarchy due to the definition of Projects as a TaskEntity subtype. This implies that projects may be decomposed into subprojects, and that they have a limited lifespan.

Task transition chains can be used to describe phases or revisions of software components at a more detailed level:

- Horizontal life-cycles, such as (Requirements, Specification, Design, Imple mentation, Testing, Release, Delivery). 
- Revision lines, such as edit, review and test operations on individual objects within a lifecycle phase. Status attributes with values such as (Initial, Experimental, .... Finished, Integrated, Approved) are suitable for composites like a configuration.

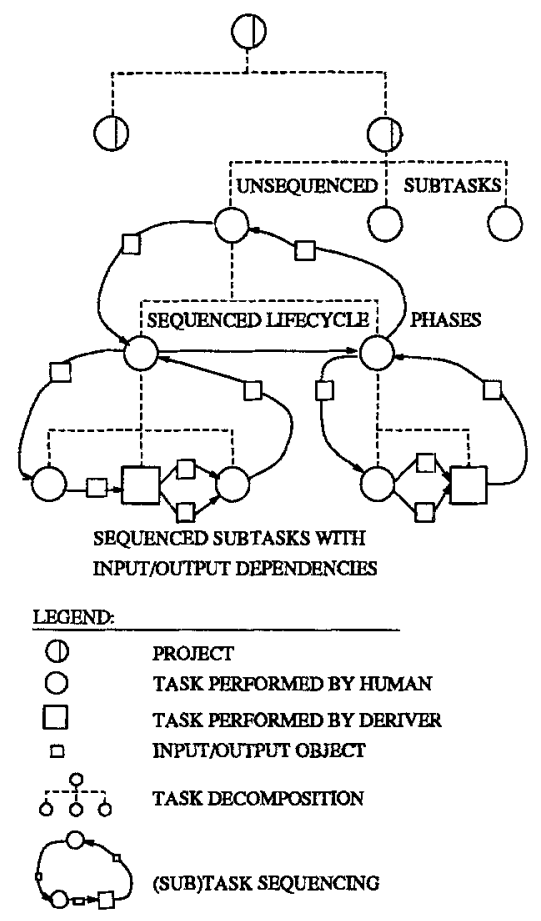

Figure 6: General Subtasking

Below is an example of task modelling of a development task, with 5 subtasks of the requirements specification phase:

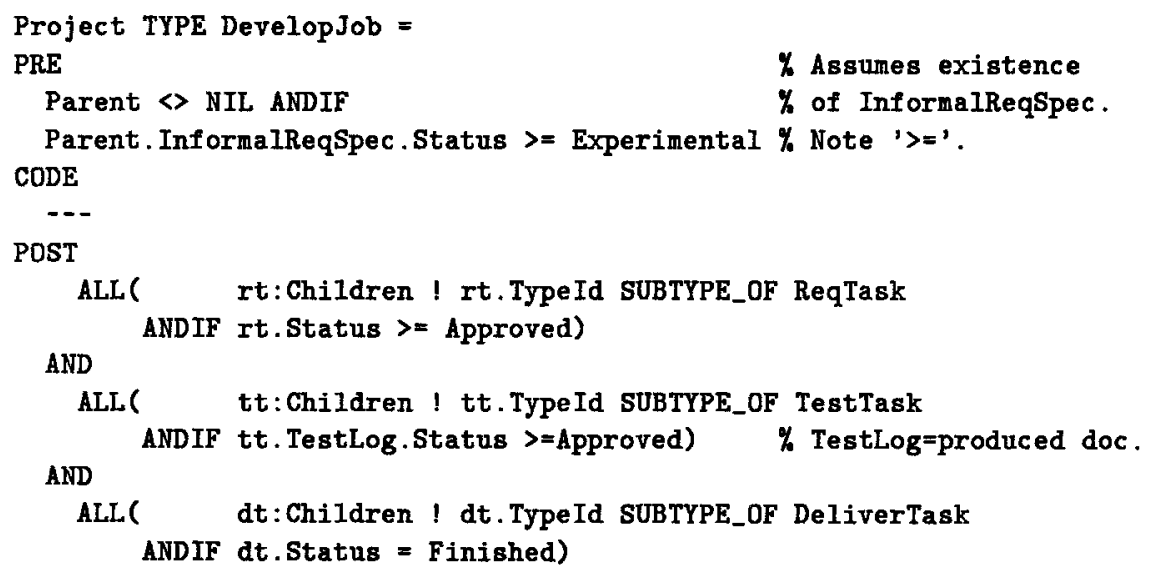




\section{Conclusion and Future Work}

The EPOS PM model provides both a dynamic and a static view of description, planning and execution of software processes. The model covers deriver tools, human actors, high-level projects and low-level tool activations, task transition networks, and project and task decomposition. Both type and instance hierarchies can be used to express task knowledge.

The CM and PM areas are connected through a common data model, EPOSOOER. CM is coupled to PM through change jobs associated with configdescriptions, and through more detailed revision tasks. Likewise, PM-relevant control information is contained in a project $\mathrm{KB}$, which is versioned (i.e. controlled by $\mathrm{CM}$ ) to allow easy project customization and evolution.

All in all, we think that the proposed PM support is a fruitful basis for continued work in the area. Still, there are many issues to be pursued:

- A more powerful, imperative CODE language.

- The well-suitedness of PRE/POST-conditions for for general task synchronization. Consider a task that can be activated in three different ways, identified by PRE-conditions B1, B2, and B3. This has to be written as:

PRE

B1 OR B2 OR B3 OR -.-

CODE

IF B1 THEN Code1

ELSEIF B2 THEN Code2

ELSEIF B3 THEN Code3

ELSE -.-;

- More high-level type templates for task/tool patterns. See e.g. [ENE87] on graph grammars.

- A more generic and possibly dynamic data model, to avoid proliferation of trivial subtypes each time e.g. a new programming language is added. This resembles versioning of task types according to the current project.

- Better formalization of projects, and their workspaces for long transactions.

- Overall methodologies for project and process modelling.

- Better modelling of CASE-like meta-tools with internal tool policies.

- Planning: heuristics, intertwined planning and execution, knowledge representation, and $\mathrm{KB}$ support.

- Industrial scenarios and trial use.

Only a prototype EPOS implementation will be built in Trondheim, and only of the basic CM and PM system. On the other hand, CM has high priority within our industrial partners, Sysdeco and Veritas Research, so that future industrialization seems assured. 


\section{Acknowledgements}

Thanks to P. Lavency et al. from Philips Research in Brussels on PM, and to J. Müller from the Techn. Univ. of Karlsruhe on the Planner design.

\section{References}

[AS88] José A. Ambros-Ingerson and Sam Steel. Integrating planning, execution and monitoring. In Proc. of AAAI'88, pages 83-88, 1988.

[B*89] K. Benali et al. Presentation of the ALF project. In Prelim. Proceedings from Int'l Conf. on SDEF, Berlin, 9-11 May 1989, page 23 p., May 1989.

[BE87] Noureddine Belkhatir and Jacky Estublier. Software management constraints and action triggering in the ADELE program database. In [NS87], pages 4454, 1987.

[BL89] Yves Bernard and Pierre Lavency. A Process-Oriented Approach to Configuration Management. In [IEE89], 1989. $14 \mathrm{p}$.

[BLA88] Roberto Bisiani, F. Lecouat, and Vinzenco Ambriola. A Planner for the automation of programming environment tasks. In Proc. of the 21st Annual Hawaii International Conference on System Sciences, pages 64-72, Hawaii, USA, January 1988.

[Bul87] PACT: The initial PACT Environment. Bull, Louveciennes, France, September 1987.

[C*89] Reidar Conradi et al. EPOS Day Compendium - 1 Nov. 1989. Technical Report, DCST, NTH, Trondheim, Norway, October 1989. NTH, 23 Oct 1989, $174 \mathrm{p}$.

[CDrG*89] Reidar Conradi, Tor Martin Didriksen, Bjørn Gulla, Håvard Eidnes, EvenAndré Karlsson, Anund Lie, Per Harald Westby, Svein Olav Hallsteinsen, Per Holager, and Ole Solberg. Design of the kernel EPOS software engineering environment. In Proc. from Int'l Conf. on System Development Environments \& Factories, Berlin, May 1989. 16 p., Rev. Oct. 1989. Forthcoming as a Springer LNCS.

[Che76] P. P.-S. Chen. The entity-relationship model - towards a unified view of data. ACM Transactions on Database Systems, 1(1):9-36, March 1976.

[Cle88] Geoffrey M. Clemm. The Odin specification language. In [Win88], pages 144$158,1988$.

[CW85] Luca Cardelli and Peter Wegner. On Understanding Types, Data Abstraction, and Polymorphism. Computing Surveys, 17(4):471-521, 1985.

[DGS89] Wolfgang Deiters, Volker Gruhn, and Wilhelm Schäfer. Systematic development of software process models. In Carlo Ghezzi, John A. McDermid (Eds.): Proc. of ESEC'89 - the 2nd European Software Engineering Conference '89, Warwick, UK, September 1989. Springer Verlag LNCS 387, p. 100-117. 
[Dow87] Mark Dowson. ISTAR and the contractual approach. In Proc. of the 9th ACM-SIGSOFT/IEEE-CS Int'l Conference on Software Engineering, Monterey, $C A, U S A$, pages 287-288, April 1987.

[ENE87] Proc. Srd Int'l Workshop on Graph Grammars and their Application to Computer Science, Warrenton, VA, USA, 1987.

[GEC87] GENOS, GEC Software's IPSE. GEC Software, London, UK, May 1987.

[HC88] Dennis Heimbigner and Steven Crane. A graph transform model for configuration management environments. In [Hen88], pages 216-225, 1988.

[Hen88] Peter B. Henderson, editor. Proc. of the Srd ACM SIGSOFT/SIGPLAN Software Engineering Symposium on Practical Software Development Environments (Boston, 28-30 Nov 1988), 257 p., November 1988. In ACM SIGPLAN Notices 24(2), Feb 1989.

[HM88] Tani Haque and Juan Montes. A Configuration Management System and more (on Alcatel's PCMS). In [Win88), pages 217-227, 1988.

[HN86] A. Nico Habermann and David Notkin. GANDALF: software development environments. IEEE Transactions of Software Engineering, SE-12(12):11171127, December 1986. (Special issue on GANDALF).

[Hol88] Per Holager. Elements of the Design of a Change Oriented Configuration Management Tool. Technical Report STF44-A88023, 95 p., ELAB, SINTEF, Trondheim, Norway, February 1988.

[IEE89] IEEE/ACM, editor. Proc. of the 11th International Conference on Software Engineering, Pittsburgh, USA, May 1989.

[KF 87] Gail E. Kaiser and Peter H. Feiler. An architecture for intelligent assistance in software development. In Proc. of the 9th ACM-SIGSOFT/IEEE-CS Int'l Conference on Software Engineering, Monterey, CA, USA, pages 180-188, April 1987. (on MARVEL).

[LC89] Chunnian Liu and Reidar Conradi. Planning Software Development Processes in EPOS. October 1989. In [C*89].

[LCD*89] Anund Lie, Reidar Conradi, Tor M. Didriksen, Even André Karlsson, Svein O. Hallsteinsen, and Per Holager. Change Oriented Versioning in a Software Engineering Database. In Proc. of 2nd Int'l Workshop on Software Configuration Management, Princeton, USA, October 1989. ACM SIGSOFT Engineering Notes, Vol. 17, Number 7 (Nov. 1989), pp. 56-65.

[LDC*89] Anund Lie, Tor M. Didriksen, Reidar Conradi, Even André Karlsson, Svein O. Hallsteinsen, and Per Holager. Change Oriented Versioning. In Carlo Ghezzi and John A. McDermid, editors, Proc. of ESEC'89 - the 2nd European Software Engineering Conference '89, Warwick, UK, pages 191-202, September 1989. Springer Verlag LNCS 387.

[Leh87] M. M. Lehman. Process models, process programming, programming support. In Proc. of the 9th ACM-SIGSOFT/IEEE-CS Int'l Conference on Software Engineering, Monterey, CA, pages 14-16, March 1987. (Response to an ICSE'9 Keynote Address by Leon Osterweil). 
[Lem86] P. Lempp. Integrated computer support in the software engineering environment EPOS - possibilities of support in system development projects. In Proc. 12th Symposium on Microprocessing and Microprogramming, Venice, pages 223-232, North-Holland, Amsterdam, September 1986.

[Lie89] Anund Lie. Outline Design of the EPOS Database. Draft, DCST, NTH, Trondheim, Norway, April 1989.

[Mey88] Bertrand Meyer. Eiffel: a language and environment for software engineering. The Journal of Systems and Software, 199-246, 1988.

[Mul89] Jürgen Müller. Process Management Using AI Planning Techniques. Technical Report 29/89, EPOS report 86, 117 p., DCST, NTH, Trondheim, Norway, June 1989. (MSc Thesis).

[NS87] Howard K. Nichols and Dan Simpson, editors. Proc. of the First European Software Engineering Conference (Strasbourg, Sep 1987), LNCS 289 Springer Verlag, 404 p., September 1987.

[OR86] Martyn A. Ould and Clive Roberts. Modelling iteration in the software process. In Mark Dowson, editor, Proc. of the Srd International Software Process Workshop, Breckenridge, Colorado, USA, November 1986.

[Rei85] Wolfgang Reisig. Petri Nets - An Introduction. Springer-Verlag, 161 p., 1985.

[Rum87] James Rumbaugh. Relations as semantics constructs in an object-oriented language. In Proc. of the ACM SIGPLAN Conference on Object-Oriented Programming Systems, Languages and Applications (OOPSLA '87), pages 466-481, Kissimmee, Florida, October 1987. In ACM SIGPLAN Notices 22(12), Dec 1987.

[Sun88] Network Software Environment: Reference Manual. Sun Microsystems, Inc., 2550 Garcia Avenue, Mountain View, CA 94043, USA, part no: 800-2095 (draft) edition, March 1988.

[SWKH76] Michael Stonebraker, E. Wong, P. Kreps, and G. Held. The design and implementation of INGRES. ACM Trans. on Database Systems, 1:189-222, 1976.

[SZ86] Andrea H. Skarra and Stanley B. Zdonik. The management of changing types in an object-oriented database. In Proc. of the ACM SIGPLAN Conference on Object-Oriented Programming Systems, Languages and Applications (OOPS$L A$ '86), pages 483-491, Portland, Oregon, 1986. In ACM SIGPLAN Notices 21(11), Nov 1986.

[TBC*88] Richard N. Taylor, Frank C. Belz, Lori A. Clarke, Leon Osterweil, Richard W. Selby, Jack C. Wileden, Alexander L. Wolf, and Michael Young. Foundations for the Arcadia environment architecture. In [Hen88], pages 1-13, 1988.

[TL82] Dionysios C. Tsichritzis and Frederick H. Lochovsky. Data Models. Prentice Hall, 343 p., 1982.

[Win88] Jürgen F. H. Winkler, editor. Proc. of the ACM Workshop on Software Version and Configuration Control (Grassau, FRG, 27-29 Jan 1988), Berichte des German Chapter of the ACM, Band 90,466 p., B. G. Teubner Verlag, Stuttgart, 1988. 